\title{
Outcomes of switching from crizotinib to alectinib in patients with advanced non-small cell lung cancer with anaplastic lymphoma kinase fusion
}

\author{
Yingying Pan ${ }^{1 \#}$, Wenjing Xiao ${ }^{2 \#}$, Feng Ye ${ }^{3}$, Huijuan Wang ${ }^{4}$, Yihong Shen ${ }^{5}$, Xinmin Yu ${ }^{6}, \mathrm{Xiao} \mathrm{Han}^{7}$, \\ Qian Chu ${ }^{8}$, Caicun Zhou ${ }^{1}$, Zhihong Zhang ${ }^{9}$, Shengxiang Ren ${ }^{1}$
}

${ }^{1}$ Department of Medical Oncology, Shanghai Pulmonary Hospital \&Thoracic Cancer Institute, Tongji University School of Medicine, Shanghai, China; ${ }^{2}$ Department of Tumor Radiotherapy, The Affiliated Hospital of Qingdao University, Qingdao, China; ${ }^{3}$ Xiamen Key Laboratory of Antitumor Drug Transformation Research, The First Affiliated Hospital of Xiamen University, Xiamen, China; ${ }^{4}$ Department of Medical Oncology, The Affiliated Cancer Hospital of Zhengzhou University, Henan Cancer Hospital, Zhengzhou, China; ${ }^{5}$ Department of Respiratory Medicine, The First Affiliated Hospital, Zhejiang University School of Medicine, Hangzhou, China; ${ }^{6}$ Department of Thoracic Oncology, Zhejiang Cancer Hospital, Hangzhou, China; ${ }^{7}$ Department of Internal Medicine-Oncology, Shandong Cancer Hospital and Institute, Shandong First Medical University and Shandong Academy of Medical Sciences, Jinan, China; ${ }^{8}$ Department of Oncology, Tongji Hospital of Tongji Medical College, Huazhong University of Science and Technology, Wuhan, China; ${ }^{9}$ Department of Respiratory Oncology, Anhui Provincial Cancer Hospital (The First Affiliated Hospital of USTC West District), Hefei, China

Contributions: (I) Conception and design: S Ren, Z Zhang; (II) Administrative support: C Zhou; (III) Provision of study materials or patients: Y Pan, W Xiao, F Ye, H Wang, Y Shen, X Yu, X Han, Q Chu, Z Zhang; (IV) Collection and assembly of data: Y Pan, W Xiao; (V) Data analysis and interpretation: Y Pan; (VI) Manuscript writing: All authors; (VII) Final approval of manuscript: All authors.

\#These authors contributed equally to this work.

Correspondence to: Dr. Caicun Zhou. Department of Medical Oncology, Shanghai Pulmonary Hospital \&Thoracic Cancer Institute, Tongji University School of Medicine, Shanghai 200433, China. Email: caicunzhoudr@163.com; Dr. Zhihong Zhang. Department of Stomatology, The First Affiliated Hospital of USTC (Anhui Provincial Hospital), Division of Life Sciences and Medicine, University of Science and Technology of China, 17 Lujiang Road, Hefei 230001, China. Email: zhangzhihong769@163.com; Dr. Shengxiang Ren. Department of Medical Oncology, Shanghai Pulmonary Hospital \&Thoracic Cancer Institute, Tongji University School of Medicine, Shanghai 200433, China. Email: harry_ren@126.com.

Background: Alectinib and crizotinib have been approved as first-line therapies for advanced non-small cell lung cancer (NSCLC) with anaplastic lymphoma kinase (ALK) gene fusion. However, the therapeutic efficacy and side effects are still largely unknown of patients who switched to next-generation ALK tyrosine kinase inhibitors (ALK-TKIs), such as alectinib, after experiencing no disease progression with initial crizotinib treatment.

Methods: This prospective real-world study enrolled patients who were treated with alectinib after experiencing no disease progression with initial crizotinib treatment. The patients' baseline characteristics, objective response rate (ORR) of crizotinib and alectinib, size change of target tumor lesions, treatment regimen and adverse events (AEs) were collected and analyzed.

Results: The study included 53 patients, the majority of whom (96.2\%) had non-squamous NSCLC. The median age was 51 (range, 31-80) years old. The ORR of first-line crizotinib was 54.7\%. The ORR of sequential alectinib was $73.6 \%$, and $90.5 \%$ of patients showed further tumor shrinkage after the alectinib treatment. The median progression-free survival was not reached, and $90.5 \%$ of patients were still enrolled in the study at the last follow-up. Among them, $34.0 \%$ of patients switched to alectinib treatment due to the toxicity. Crizotinib was associated with a higher frequency of AEs of grades 3 and 4 than alectinib (15.1\% vs. $0 \%)$. Neither group had any AEs resulting in death.

Conclusions: Switching to alectinib might be an option for patients who do not experience disease progression with initial crizotinib therapy, and may promote better treatment compliance.

Keywords: ALK fusion; non-small cell lung cancer (NSCLC); crizotinib; alectinib 
Submitted May 04, 2021. Accepted for publication Jun 16, 2021.

doi: 10.21037/atm-21-2769

View this article at: https://dx.doi.org/10.21037/atm-21-2769

\section{Introduction}

Lung cancer therapy has entered into the era of precision medicine. For patients harboring driver oncogenes, molecular targeted therapies have unlocked a dramatic improvement in survival. The anaplastic lymphoma kinase (ALK) is a receptor tyrosine kinase encoded by the ALK gene and is often associated with lung cancer when mutated. This kinase is typically expressed in the central and peripheral nervous systems (1). ALK is reported to regulate several different pathways involved in cellular proliferation and survival, such as PI3K-AKT-mTOR, RAS-RAF-MEKERK, and the JAK-STAT pathway, once it dimerizes and is activated by autophosphorylation after binding with its ligands, pleiotrophin (PTN), and midkine (MK) $(2,3)$. For the population of patients with advanced non-small cell lung cancer (NSCLC) with anaplastic lymphoma kinase (ALK) fusion, which comprises $3 \%$ to $13 \%$ of NSCLC cases) (4), approval has been granted for the use of several first- or second-generation ALK-tyrosine kinase inhibitors (ALK-TKI), including crizotinib, ceritinib, alectinib, and brigatinib $(5,6)$.

Alectinib and brigatinib were recommended by the National Comprehensive Cancer Network guidelines (version 1, 2021) as the preferred first-line therapies for advanced NSCLC with ALK fusion (7). Ceritinib, alectinib, and brigatinib have also been approved as the second-line therapies to treat crizotinib-resistant ALK fusion non-small cell lung cancer (NSCLC) (7-9). In a matching-adjusted indirect comparison (MAIC) conducted to estimate the relative efficacy of these agents, the median progressionfree survival (PFS) with ceritinib, alectinib, and brigatinib ranged between 6.9 and 7.2 months, 8.2 and 8.9 months, and 16.7 months, respectively (10). In the United States, lorlatinib recently received approval as a treatment for patients whose disease has progressed on crizotinib and one additional ALK-TKI therapy or after failure of alectinib or ceritinib treatment $(11,12)$.

Second-generation ALK inhibitors, such as alectinib, are able to stay at higher concentrations in the central nervous system (CNS) (13). They can also attain longer PFS and overall survival (OS), as well as fewer side effects, than crizotinib in the first-line setting for advanced NSCLC with ALK fusion (14). However, access to these novel drugs is still largely limited mainly because it depends on approval and reimbursement decisions. For instance, a recent survey showed that crizotinib still being the main treatment as alectinib is still not available in the majority of central European countries because of a long lag interval between EMA or national MA and national reimbursement decisions (15). Therefore, we will inevitably encounter the situation that next-generation ALK-TKIs such as alectinib are available for these patients who did still not progress from the initial treatment of crizotinib after alectinib was approval. However, the therapeutic efficacy and side effects of patients who switch to next-generation ALKTKI without crizotinib-refractory after initial crizotinib treatment are still largely unknown.

In this study, we prospectively collected 53 patients with ALK-fusion NSCLC whose initial crizotinib treatment did not fail and subsequently received alectinib as following therapy. We observed further significant tumor shrinkage and better side effects after the alectinib treatment, which suggests that the strategy of switching to alectinib might be a therapeutic option for patients whose disease does not progress from initial treatment with crizotinib.

We present the following article in accordance with the STROBE reporting checklist (available at https://dx.doi. org/10.21037/atm-21-2769).

\section{Methods}

\section{Patients enrollment}

Patients with $A L K$-fusion NSCLC who received the ALK-TKI crizotinib as a first-line treatment followed by alectinib without showing crizotinib resistance were enrolled from the following Chinese institutions between September 2015 and March 2020: "The Department of Medical Oncology, Shanghai Pulmonary Hospital", "The First Affiliated Hospital of Xiamen University", "Department of Respiratory Oncology, Anhui Provincial Cancer Hospital (The First Affiliated Hospital of USTC West District)", "Department of Oncology, The Affiliated Hospital of Qingdao University", "Department of Respiratory Medicine, The First Affiliated Hospital, 
Zhejiang University School of Medicine", "Department of Thoracic Oncology, Zhejiang Cancer Hospital", "Department of Medical Oncology, The Affiliated Cancer Hospital of Zhengzhou University, Henan Cancer Hospital", "Department of Internal Medicine-Oncology, Shandong Cancer Hospital and Institute", and "Tongji Medical College of HUST, Tongji Hospital". All patients had a pathological diagnosis of lung cancer based on the World Health Organization (WHO) classification (16). Patients' clinicopathological information was obtained from their medical records, which were available electronically from the institutions. All patients had chosen to switch crizotinib to alectinib therapy before crizotinib resistance developed, and carried on with the alectinib therapy until disease progressed [according to the Response Evaluation Criteria in Solid Tumors (RECIST), version 1.1] (17), or experienced unbearable toxicity, withdrew consent, or died of any reason. All procedures performed in this study involving human participants were in accordance with the Declaration of Helsinki (as revised in 2013). The study does not require ethical approval, as human blood and histological specimens were not involved in the study. Prior to receiving any treatments, all participants gave written informed consent for their clinical information to be used for research purposes.

\section{Assessments}

Each patient underwent computed tomography (CT) examination. Tumors were evaluated based on RECIST 1.1. Size changes of target tumor lesions were calculated as relative changes in the sum of the target lesions based on the investigator's measurements.

The efficacy of alectinib was determined by comparing the patients' situation when their therapy was switched to alectinib with their situation after alectinib treatment. The ORR was taken as the percentage of patients who had a complete response (CR) or partial response (PR), as determined by RECIST 1.1. The disease control rate (DCR) was calculated on the basis of percentages of patients with $\mathrm{CR}, \mathrm{PR}$, and stable disease after treatment according to RECIST 1.1. The National Cancer Institute's Common Terminology Criteria for Adverse Events version 4.03 was used for the classification and grading of adverse events (AEs).

ALK-fusion analyses were performed at the different hospitals. Patients' ALK status was determined on the basis of fluorescence in situ hybridization (FISH), reverse transcription PCR, immunohistochemistry (IHC), or nextgeneration sequencing (NGS). A diagnosis of ALK-fusion NSCLC was confirmed by a positive result for any of these tests (18).

\section{Statistical analyses}

Standard descriptive statistics were used to analyze patients' demographic and clinical information at baseline. Frequencies and percentages were used to describe categorical variables. SPSS version 22.0 Software (SPSS, Inc., Chicago, IL) was employed to perform the statistical analysis. Excel (Microsoft 2016) and R studio were used to create the figures.

\section{Results}

\section{Patients' baseline characteristics}

Fifty-three patients with ALK-fusion NSCLC whose disease was measurable with RECIST criteria 1.1 at baseline were identified. The study participants had a median age of 51 (range, 31-80) years old, and females accounted for $62.3 \%$ of the cohort. Among the participants, $88.7 \%$ had never smoked. Most cases (96.2\%) were histologically classified as non-squamous NSCLC. In $98.1 \%$ of cases, the Eastern Cooperative Oncology Group performance status score was 0 or 1 . Stage III and IV disease accounted for $33.9 \%$ and $60.4 \%$ of cases, respectively. Of the patients, $58.5 \%$ had intrathoracic metastasis and $77.4 \%$ had extrathoracic metastasis. Table 1 shows the characteristic information of the study participants. All participants received crizotinib as first-line treatment and then switch to alectinib after experiencing no disease progression from crizotinib.

Regarding ALK status testing, $35.8 \%$ of patients were tested by NGS, $35.9 \%$ of patients tested positive with IHC [VENTANA anti-ALK (D5F3) rabbit monoclonal primary antibody, Roche], and $24.5 \%$ were tested using PCR. FISH was used in only $2(3.8 \%)$ cases in our study.

All patients in the cohort took crizotinib as a first-line ALK-TKI without disease progression. Of the patients, $34.0 \%$ changed the treatment to alectinib due to crizotinib intolerance, and $28.3 \%$ changed because their best response to crizotinib was stable disease, and they wanted to seek a more efficacious treatment. The other $37.7 \%$ of patients changed treatment for other reason, of note, alectinib was officially approved for coverage under Chinese Medical 
Table 1 Baseline characteristics of the patients

\begin{tabular}{|c|c|}
\hline Baseline characteristics of the patients & Value \\
\hline Median age at diagnosis, years [range] & $51[31-80]$ \\
\hline \multicolumn{2}{|l|}{ Sex, n (\%) } \\
\hline Male & $20(37.7)$ \\
\hline Female & $33(62.3)$ \\
\hline \multicolumn{2}{|l|}{ Smoking history, n (\%) } \\
\hline Never-smoker & $47(88.7)$ \\
\hline Ever-smoker & $6(11.3)$ \\
\hline \multicolumn{2}{|l|}{ ECOG PS, n (\%) } \\
\hline 0 & $16(30.2)$ \\
\hline 1 & $36(67.9)$ \\
\hline 2 & $1(1.9)$ \\
\hline \multicolumn{2}{|l|}{ Histologic type, n (\%) } \\
\hline Non-squamous & $51(96.2)$ \\
\hline Squamous & $2(3.8)$ \\
\hline \multicolumn{2}{|l|}{ Stage at initial diagnosis, $\mathrm{n}(\%)$} \\
\hline Stage I & $2(3.8)$ \\
\hline Stage II & $1(1.9)$ \\
\hline Stage III & $18(33.9)$ \\
\hline Stage IV & $32(60.4)$ \\
\hline \multicolumn{2}{|l|}{ Baseline metastasis, n (\%) } \\
\hline Intrathoracic & $31(58.5)$ \\
\hline Extrathoracic & $41(77.4)$ \\
\hline \multicolumn{2}{|l|}{ Method of ALK testing, $n(\%)$} \\
\hline Pts with NGS results & $19(35.8)$ \\
\hline Pts with FISH results & $2(3.8)$ \\
\hline Pts with IHC Ventana results & $19(35.9)$ \\
\hline Pts with PCR results & $13(24.5)$ \\
\hline \multicolumn{2}{|l|}{ Initial disease status, n (\%) } \\
\hline Yes & $10(18.9)$ \\
\hline No & $43(81.1)$ \\
\hline \multicolumn{2}{|l|}{ Reason for treatment change, $\mathrm{n}(\%)$} \\
\hline Crizotinib intolerance & $18(34.0)$ \\
\hline Best curative effect SD & $15(28.3)$ \\
\hline Other & $20(37.7)$ \\
\hline
\end{tabular}

ALK, anaplastic lymphoma kinase; ECOG, Eastern Cooperative Oncology Group; NGS, next-generation sequencing; FISH, fluorescence in-situ hybridization; SD, stable disease; Pts, patients.
Table 2 Summary of the patients' treatment response data

\begin{tabular}{lcc}
\hline Best response & Crizotinib $(n=53)(\%)$ & Alectinib $(n=53)(\%)$ \\
\hline CR & $0(0.0)$ & $0(0.0)$ \\
PR & $29(54.7)$ & $39(73.6)$ \\
SD & $24(45.2)$ & $14(26.4)$ \\
PD & $0(0.0)$ & $0(0.0)$ \\
ORR & $29(54.7)$ & $39(73.6)$ \\
DCR & $53(100.0)$ & $53(100.0)$ \\
\hline CR, complete response; PR, partial response; SD, stable \\
disease; PD, progressive disease; ORR, objective response rate; \\
DCR, disease control rate.
\end{tabular}

Insurance in 2020, and the price of alectinib decreased to a considerable extent as a result.

\section{Therapeutic responses to ALK-TKI treatment in patients}

In the study cohort, the ORR of first-line crizotinib was $54.7 \%$, and the DCR reached $100 \%$ (Table 2). The ORR and DCR of sequential alectinib were $73.6 \%$ and $100 \%$, respectively (Table 2 and Figure 1), which showed no statistical decrease from the rates previously reported (14). The last follow-up date was November 30, 2020; at this point, all of the patients had switched to receive alectinib as sequential therapy, and their responses to this treatment were good. The treatment regimen of each patient is shown in Figure 2.

Figure 3 shows the overall response of the patients. In the majority of cases, the disease response continued, which suggests that switching to alectinib after taking crizotinib without disease progression may not influence the efficacy of alectinib. Figure 4 shows representative CT images of one patient in the study.

\section{Systemic AEs of ALK-TKI treatment in patients}

We further recorded the AEs of crizotinib and alectinib in patients. Table 3 and Figure 5 show all-cause AEs reported in the patients during the course of either treatment. Elevated transaminase levels (22.6\%), vomiting (22.6\%), and visual disorders $(18.9 \%)$ occurred more frequently with crizotinib, whereas constipation ( $45.3 \%)$, edema $(41.5 \%)$, and skin toxicities $(26.4 \%)$ were reported more frequently with alectinib. Of the all-cause AEs of grades 3 and 4 with crizotinib, increased transaminase levels (5.7\%) 


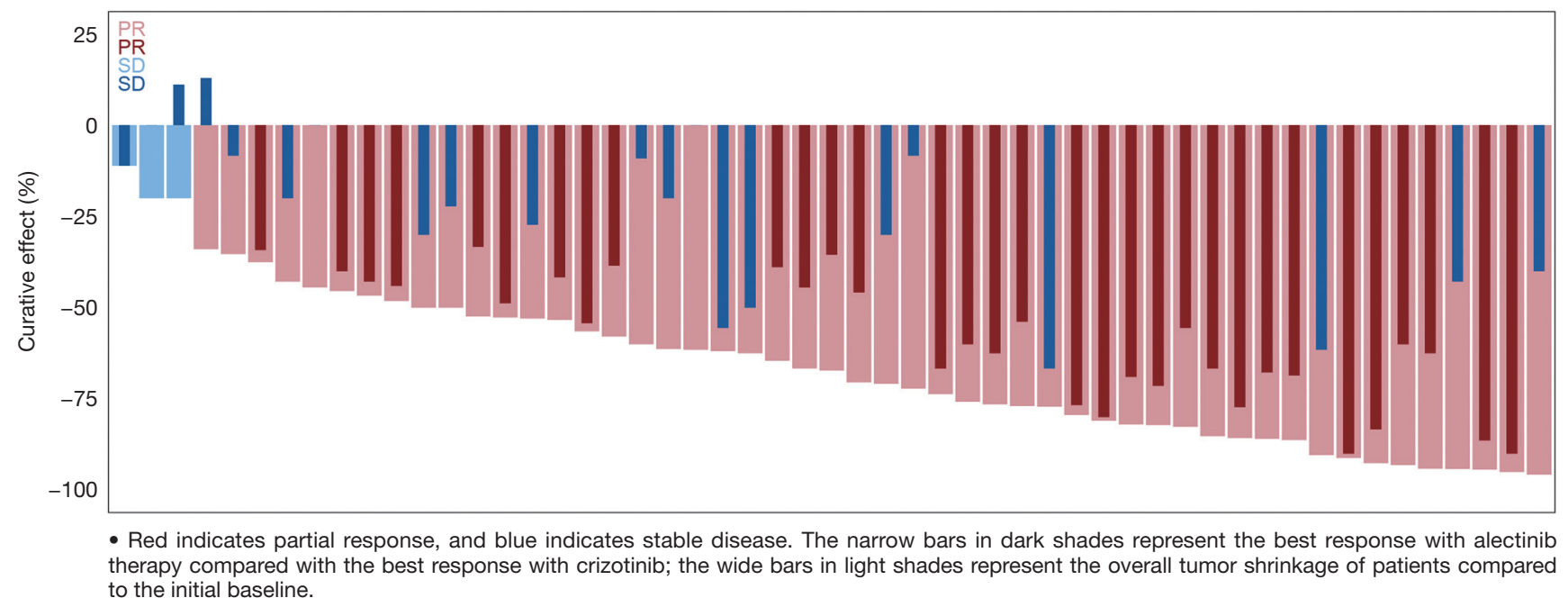

Figure 1 Best radiographic change (\%) in the target lesions of each patient.

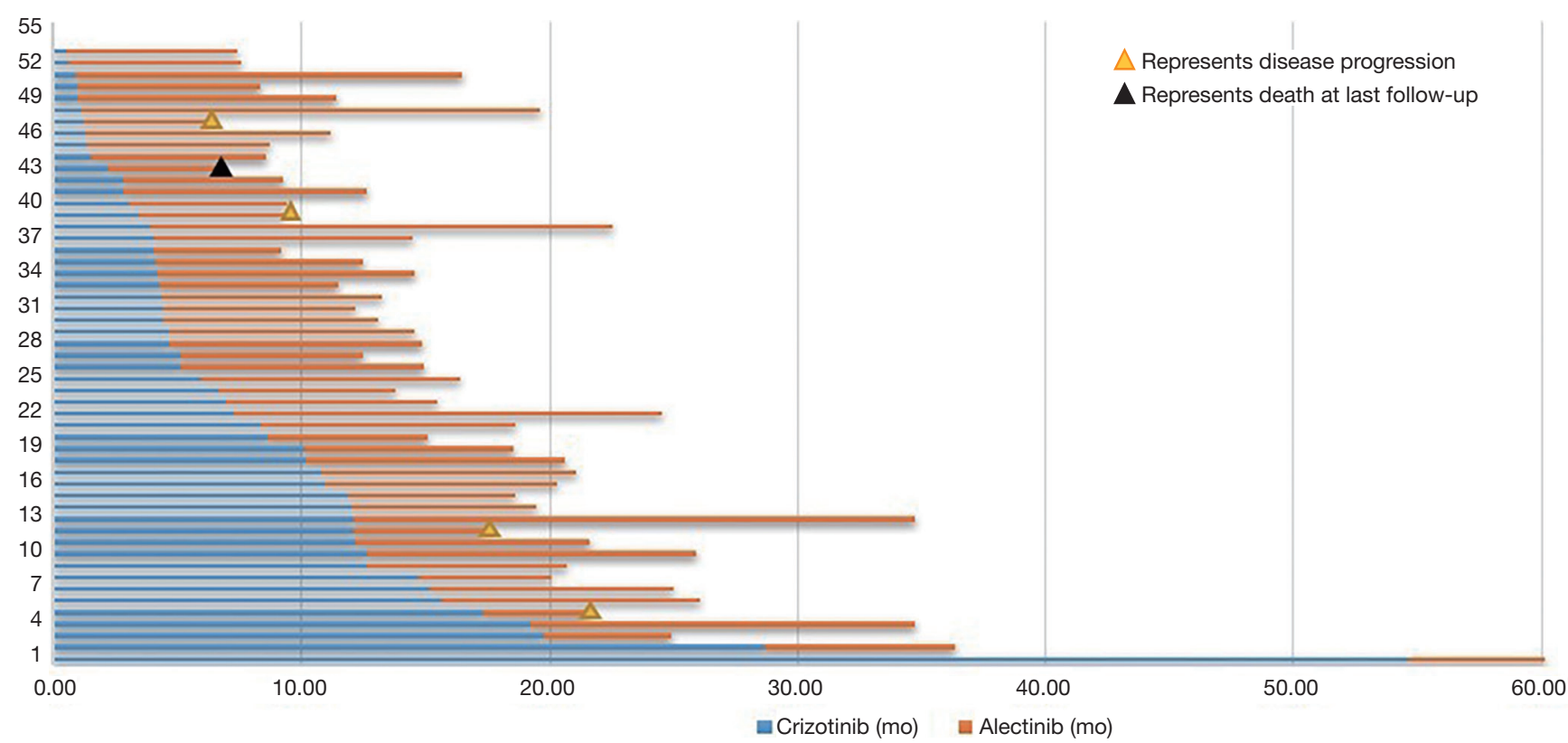

Figure 2 Treatment times of crizotinib and sequential alectinib in the patients with ALK-fusion non-small-cell lung cancer.

and dizziness $(1.9 \%)$ occurred most frequently. No AEs of grades 3 or 4 have been reported with alectinib among the study participants so far.

\section{Discussion}

To the best of our knowledge, the present work is the first investigation of alectinib's efficacy and side effects in patients with advanced NSCLC with ALK fusion who experienced no disease progression after initial treatment with crizotinib. We respectively enrolled 53 patients with ALK-fusion NSCLC and observed an ORR of $73.6 \%$. Furthermore, $90.5 \%$ of patients had further tumor shrinkage after receiving alectinib. We also observed significantly fewer side effects among the patients after treatment with alectinib compared with crizotinib. At the last-follow up, $90.5 \%$ of the study participants were still receiving alectinib treatment, which suggests that the 


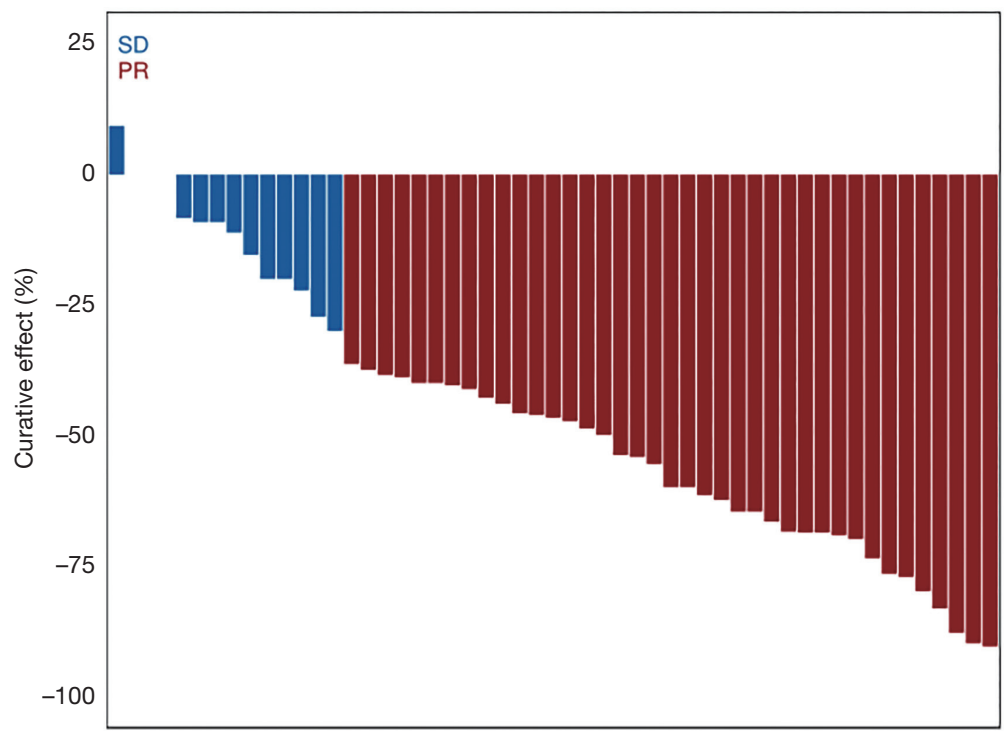

Figure 3 Tumor shrinkage with alectinib best response compare to the tumor size at the timepoint of switching treatment.

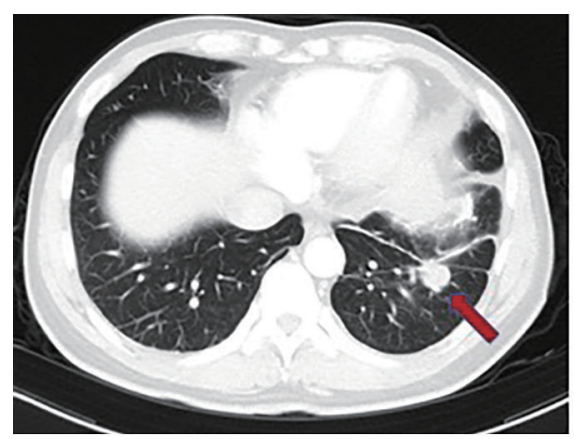

CRZ baseline

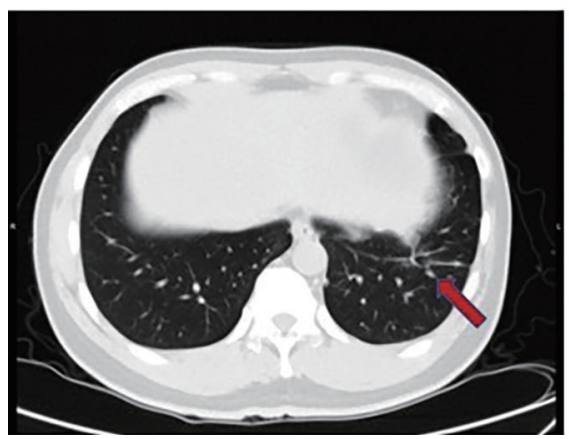

First assessment of ALE

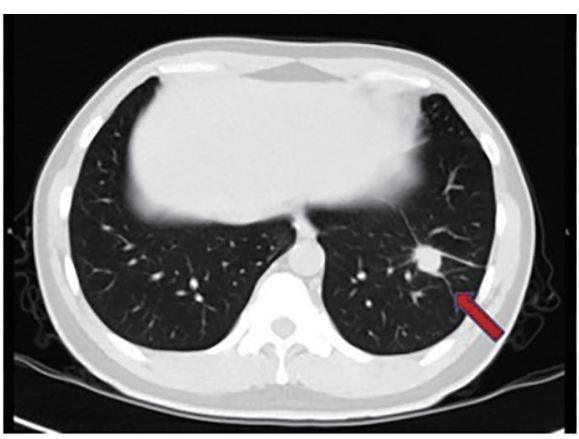

First assessment of $\mathrm{CRZ}$

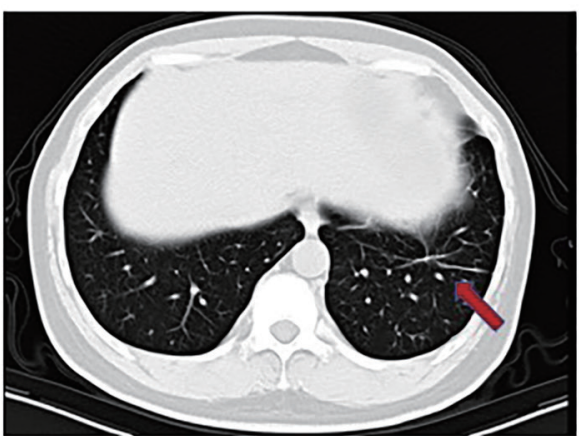

Follow up at 6 mo

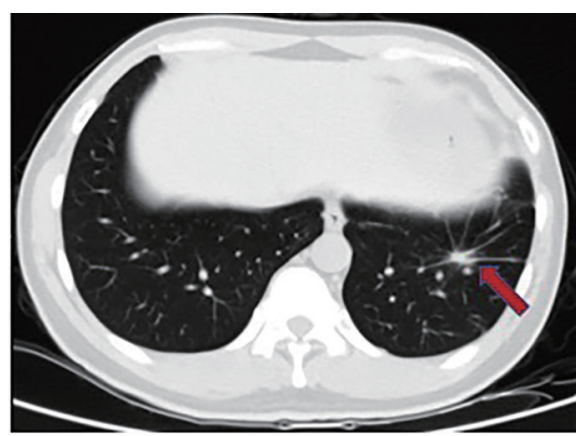

Baseline of switching to ALE

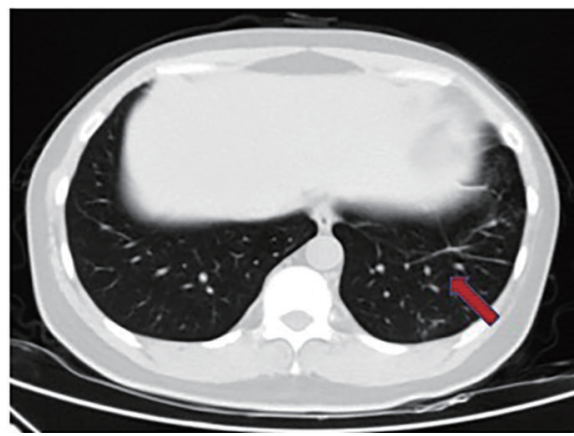

Follow up at $12 \mathrm{mo}$

Figure 4 Representative CT images of a patient. The red arrows indicate the target lesion of tumor. ALE, alectinib; CRZ, crizotinib; mo, month.

strategy of switching from crizotinib to alectinib could be an alternative regimen for patients with advanced ALK fusion NSCLC whose initial crizotinib treatment has not failed.
Recently, a dramatic change has occurred in the treatment of patients with advanced NSCLC and ALK fusion. Crizotinib is a first-generation ALK-TKI which shows a superior ORR and PFS compared with standard 
Table 3 Adverse events reported in patients in during the course of either treatment

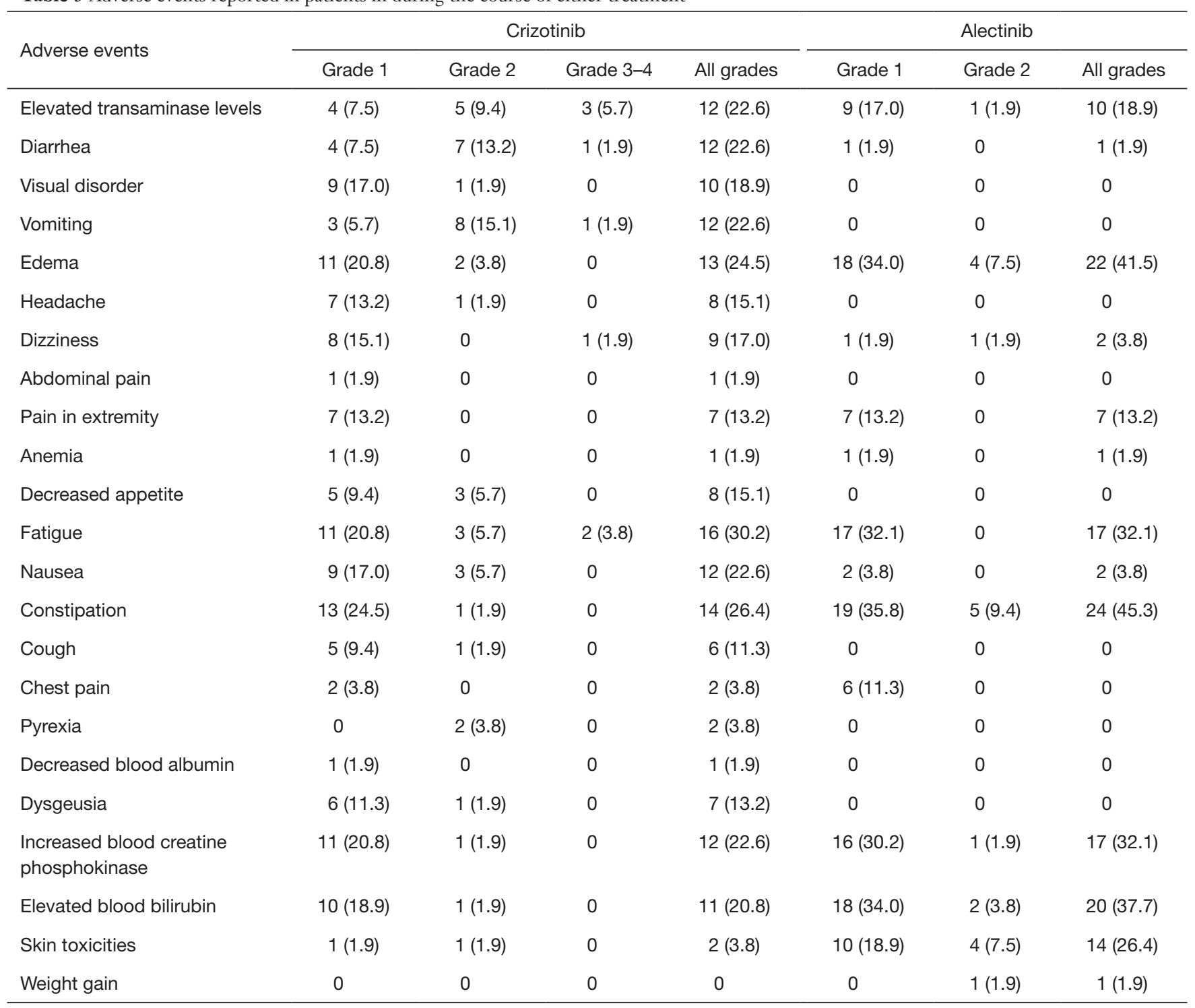

Data represent the number of patients with at least 1 event (\% of total patients).

chemotherapeutic regimens for advanced disease in the first- and second-line settings $(19,20)$. However, as many pre-clinical and clinical models have suggested, most patients develop resistance to crizotinib through various mechanisms within a year $(21,22)$, particularly in the CNS because of insufficient penetration of the blood-brain barrier $(23,24)$. Secondary mutations in the ALK gene are considered to be the most frequent mechanisms mediating resistance to ALK inhibitors which render crizotinib less effective by decreasing ligand affinity for its active site $(18,25)$. To overcome these shortcomings, several second or third generation ALK inhibitors, such as ceritinib (8), alectinib, brigatinib (7), and lorlatinib (11), have been developed with the purpose of prolonging the disease control and managing CNS localization.

Alectinib, a powerful and selective, Adenosine triphosphate (ATP)-competitive, second-generation TKI, whose main activity is targeting ALK fusion and rearranged during transfection (RET) gene rearrangements (26). Upon discovery, crizotinib targets MET, ROS1 and ALK. Crizotinib and ceritinib are both targets of p-glycoprotein (P-gp), a membrane protein that pumps xenobiotics out 


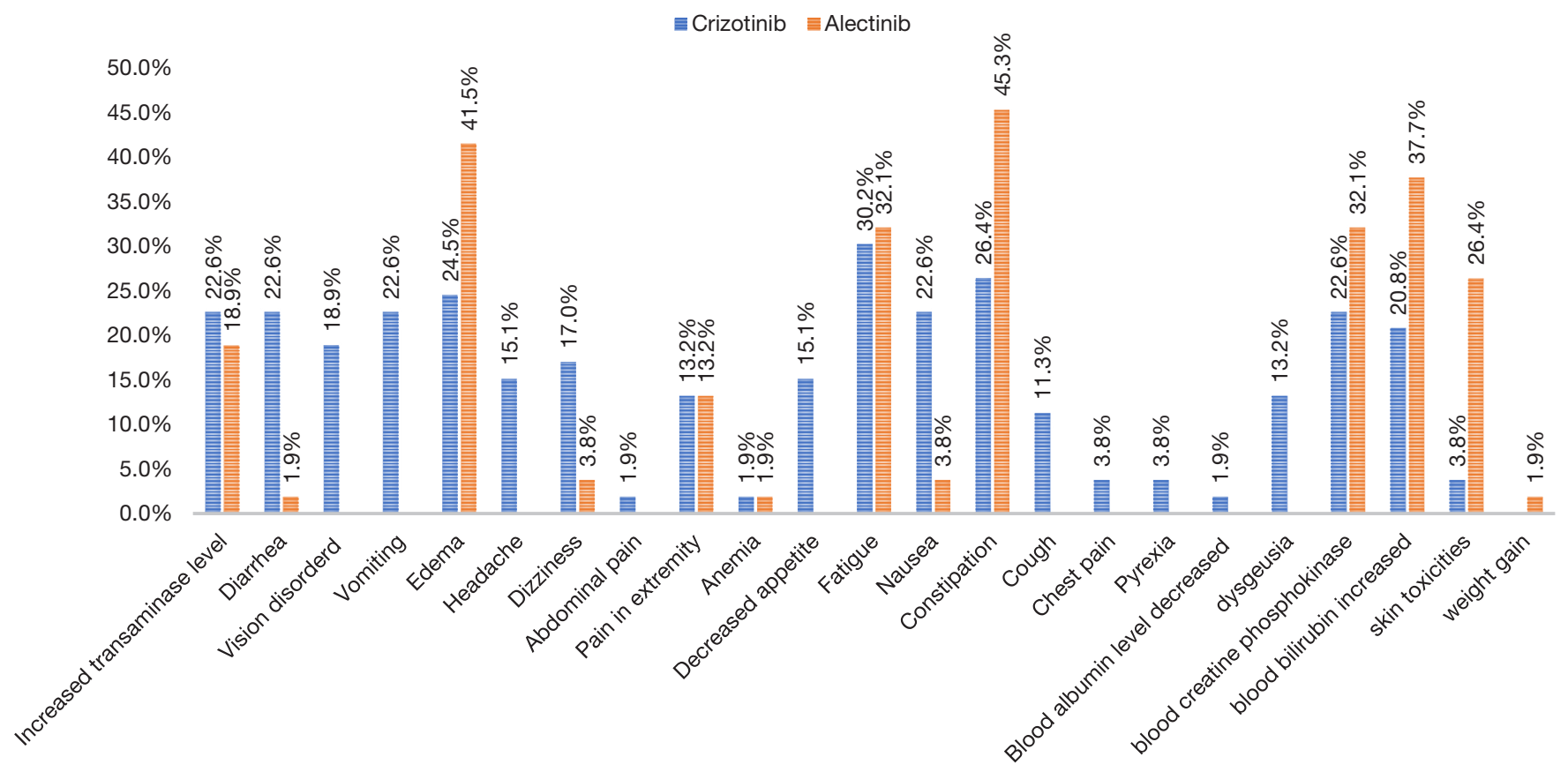

Figure 5 All-grade treatment-related adverse events of crizotinib and alectinib.

of the central nervous system (CNS), whereas alectinib is not (27). For this reason, the brain is a common site of relapse in patients treated with crizotinib (28). Unlike crizotinib, alectinib is a CNS penetrant; it is not a substrate of P-glycoprotein, a key efflux transporter located at the blood-brain barrier (29). With strong CNS penetration ability, alectinib was demonstrated to be a growth inhibitor of ALK fusion CNS lesions in an intracranial tumor xenograft model (30). Furthermore, 3 phase III trials $(9,14,31)$ produced consistent evidence that alectinib is superior to crizotinib alone with respect to PFS, OS, and toxicity in the first-line setting, with the median PFS reaching 34.1 and 34.8 months in the J-ALEX (number: JapicCTI-132316) and ALEX (number: NCT02075840) trials, respectively. Therefore, alectinib was recommended as one of the preferred options over crizotinib in the latest NCCN guidelines.

Despite the tremendous changes that have been achieved for patients with lung cancer, the worldwide availability of novel anti-cancer drugs is often delayed. Alectinib, for instance, was first approved in September 2018; however, only in January 2020 was it approved for coverage under the health insurance reimbursement system in China. Moreover, a recent survey showed that alectinib is still not available in majority of countries in central Europe (15). Therefore, patients with ALK-fusion NSCLC will inevitably face the situation that initial treatment resistance of crizotinib and simultaneously had the availability of more potent secondgeneration ALK-TKI as sequential therapy. Our study is the first investigation of alectinib's efficacy and side effects in patients with advanced NSCLC with ALK fusion for whom initial treatment with crizotinib did not fail. We observed that $90.5 \%$ of patients experienced further tumor shrinkage after using alectinib, which indicates that the residual tumors are still dependent on the ALK pathway (32).

Several second-line trials of alectinib have shown that some patients can experience tumor shrinkage after crizotinib failure (33). For example, in the phase III clinical trial ALUR (number: NCT02604342), the investigatorassessed ORR (Intention-To-Treat, ITT) of alectinib after crizotinib failure was $37.5 \%$ (27/72 patients) (34). Further, WJOG9516L (35) reported that the ORR of sequential alectinib was $35.6 \%$ in the progressive-disease subgroup of patients who had received crizotinib, which indicates that crizotinib does not completely inhibit the ALK signal.

Reducing the occurrence of systemic AEs constitutes a central aspect of individualized medicine and targeted therapy. In our study, gastrointestinal AEs and increased transaminase levels were the highest-occurring AEs, and severe gastrointestinal AEs significantly decreased patient 
compliance. Alectinib is viewed as one of the most tolerable ALK-TKIs, as a result, it is rarely refused by patients (36). In this study, $34.0 \%$ of patients switched to alectinib because of crizotinib intolerance. After treatment switching, the enrolled patients displayed significantly fewer side effects such as nausea/vomiting, which further supports the strategy of switching treatments used in this study.

There were several limitations to this study that should be mentioned. Firstly, due to the longer median PFS with first-line alectinib treatment, the observation period was not sufficient to reach the median PFS and OS, and therefore, AEs experienced by the patients may not have been fully reported. Secondly, the number of patients enrolled in this study was limited. Therefore, selection bias cannot be ignored and might be responsible for the favorable outcomes. Thirdly, due to medical insurance and reimbursement policies, the use of alectinib is more widespread in China and fewer patients receive crizotinib in the first-line setting than before, which also stresses the importance of the current study.

In conclusion, patients with ALK-fusion NSCLC who received initial crizotinib treatment without progression disease exhibited significant tumor shrinkage and less severe side effects after switching to alectinib therapy. Our observations suggest that switching from crizotinib to alectinib could be an alternative treatment strategy for patients with advanced NSCLC with ALK fusion. We expect that the long-term PFS and OS follow-up data of these patients will further support our conclusions.

\section{Acknowledgments}

Funding: This work was supported by the National Natural Science Foundation of China (No. 81772467 to SR, No. 81972167 to SR), Shanghai Shenkang Hospital Development Center (No. SHDC12019133 to SR) and Shanghai Innovative Collaboration Project (No. 2020CXJQ02 to CZ).

\section{Footnote}

Reporting Checklist: The authors have completed the STROBE reporting checklist. Available at https://dx.doi. org/10.21037/atm-21-2769

Data Sharing Statement: Available at https://dx.doi. org/10.21037/atm-21-2769
Conflicts of Interest: All authors have completed the ICMJE uniform disclosure form (available at https://dx.doi. org/10.21037/atm-21-2769). YP, CZ, SR report that this work was supported by the National Natural Science Foundation of China (No. 81772467 to SR, No. 81972167 to SR), Shanghai Shenkang Hospital Development Center (No. SHDC12019133 to SR) and Shanghai Innovative Collaboration Project (No. 2020CXJQ02 to CZ). The other authors have no conflicts of interest to declare.

Ethical Statement: The authors are accountable for all aspects of the work in ensuring that questions related to the accuracy or integrity of any part of the work are appropriately investigated and resolved. All procedures performed in this study involving human participants were in accordance with the Declaration of Helsinki (as revised in 2013). The study does not require ethical approval, as human blood and histological specimens were not involved in the study. Prior to receiving any treatments, all participants gave written informed consent for their clinical information to be used for research purposes.

Open Access Statement: This is an Open Access article distributed in accordance with the Creative Commons Attribution-NonCommercial-NoDerivs 4.0 International License (CC BY-NC-ND 4.0), which permits the noncommercial replication and distribution of the article with the strict proviso that no changes or edits are made and the original work is properly cited (including links to both the formal publication through the relevant DOI and the license). See: https://creativecommons.org/licenses/by-nc-nd/4.0/.

\section{References}

1. Wellstein A. ALK receptor activation, ligands and therapeutic targeting in glioblastoma and in other cancers. Front Oncol 2012;2:192.

2. Morales La Madrid A, Campbell N, Smith S, et al. Targeting ALK: a promising strategy for the treatment of non-small cell lung cancer, non-Hodgkin's lymphoma, and neuroblastoma. Target Oncol 2012;7:199-210.

3. Shaw AT, Solomon B. Targeting anaplastic lymphoma kinase in lung cancer. Clin Cancer Res 2011;17:2081-6.

4. Wu J, Savooji J, Liu D. Second- and third-generation ALK inhibitors for non-small cell lung cancer. J Hematol Oncol 2016;9:19.

5. Sullivan I, Planchard D. Treatment modalities for 


\section{Page 10 of 11}

advanced ALK-rearranged non-small-cell lung cancer.

Future Oncol 2016;12:945-61.

6. Sgambato A, Casaluce F, Maione P, et al. Targeted therapies in non-small cell lung cancer: a focus on ALK/ ROS1 tyrosine kinase inhibitors. Expert Rev Anticancer Ther 2018;18:71-80.

7. Camidge DR, Kim HR, Ahn MJ, et al. Brigatinib Versus Crizotinib in Advanced ALK Inhibitor-Naive ALKPositive Non-Small Cell Lung Cancer: Second Interim Analysis of the Phase III ALTA-1L Trial. J Clin Oncol 2020;38:3592-603.

8. Shaw AT, Kim TM, Crinò L, et al. Ceritinib versus chemotherapy in patients with ALK-rearranged nonsmall-cell lung cancer previously given chemotherapy and crizotinib (ASCEND-5): a randomised, controlled, openlabel, phase 3 trial. Lancet Oncol 2017;18:874-86.

9. Hida T, Nokihara H, Kondo M, et al. Alectinib versus crizotinib in patients with ALK-positive non-small-cell lung cancer (J-ALEX): an open-label, randomised phase 3 trial. Lancet 2017;390:29-39.

10. Reckamp K, Lin HM, Huang J, et al. Comparative efficacy of brigatinib versus ceritinib and alectinib in patients with crizotinib-refractory anaplastic lymphoma kinasepositive non-small cell lung cancer. Curr Med Res Opin 2019;35:569-76.

11. Waqar SN, Morgensztern D. Lorlatinib: a newgeneration drug for ALK-positive NSCLC. Lancet Oncol 2018;19:1555-7.

12. Kuang S, Leighl NB. Lorlatinib in ALK-Rearranged Lung Cancer. Cancer Cell 2021;39:25-7.

13. Gadgeel S, Peters S, Mok T, et al. Alectinib versus crizotinib in treatment-naive anaplastic lymphoma kinase-positive (ALK+) non-small-cell lung cancer: CNS efficacy results from the ALEX study. Ann Oncol 2018;29:2214-22.

14. Camidge DR, Dziadziuszko R, Peters S, et al. Updated Efficacy and Safety Data and Impact of the EML4-ALK Fusion Variant on the Efficacy of Alectinib in Untreated ALK-Positive Advanced Non-Small Cell Lung Cancer in the Global Phase III ALEX Study. J Thorac Oncol 2019;14:1233-43.

15. Cufer T, Ciuleanu TE, Berzinec P, et al. Access to Novel Drugs for Non-Small Cell Lung Cancer in Central and Southeastern Europe: A Central European Cooperative Oncology Group Analysis. Oncologist 2020;25:e598-601.

16. Travis WD, Brambilla E, Noguchi M, et al. International association for the study of lung cancer/american thoracic society/european respiratory society international multidisciplinary classification of lung adenocarcinoma. J
Pan et al. Alectinib may benefit patient without crizotinib-resistance

Thorac Oncol 2011;6:244-85.

17. Eisenhauer EA, Therasse P, Bogaerts J, et al. New response evaluation criteria in solid tumours: revised RECIST guideline (version 1.1). Eur J Cancer 2009;45:228-47.

18. Golding B, Luu A, Jones R, et al. The function and therapeutic targeting of anaplastic lymphoma kinase (ALK) in non-small cell lung cancer (NSCLC). Mol Cancer 2018;17:52.

19. Shaw AT, Kim DW, Nakagawa K, et al. Crizotinib versus chemotherapy in advanced ALK-positive lung cancer. $\mathrm{N}$ Engl J Med 2013;368:2385-94.

20. Solomon BJ, Mok T, Kim DW, et al. First-line crizotinib versus chemotherapy in ALK-positive lung cancer. N Engl J Med 2014;371:2167-77.

21. Dagogo-Jack I, Shaw AT. Crizotinib resistance: implications for therapeutic strategies. Ann Oncol 2016;27 Suppl 3:iii42-50.

22. Isozaki H, Takigawa N, Kiura K. Mechanisms of Acquired Resistance to ALK Inhibitors and the Rationale for Treating ALK-positive Lung Cancer. Cancers (Basel) 2015;7:763-83.

23. Costa DB, Shaw AT, Ou SH, et al. Clinical Experience With Crizotinib in Patients With Advanced ALKRearranged Non-Small-Cell Lung Cancer and Brain Metastases. J Clin Oncol 2015;33:1881-8.

24. Ou SH, Jänne PA, Bartlett $\mathrm{CH}$, et al. Clinical benefit of continuing ALK inhibition with crizotinib beyond initial disease progression in patients with advanced ALKpositive NSCLC. Ann Oncol 2014;25:415-22.

25. Katayama R, Shaw AT, Khan TM, et al. Mechanisms of acquired crizotinib resistance in ALK-rearranged lung Cancers. Sci Transl Med 2012;4:120ra17.

26. Kodama T, Tsukaguchi T, Satoh Y, et al. Abstract 773: Alectinib shows potent antitumor activity against both ALK- and RET-rearranged non-small cell lung cancers. Cancer Res 2015;75:Abstract nr 773. doi: 10.1158/15387445.AM2015-773.

27. Katayama R, Lovly CM, Shaw AT. Therapeutic targeting of anaplastic lymphoma kinase in lung cancer: a paradigm for precision cancer medicine. Clin Cancer Res 2015;21:2227-35.

28. Gadgeel SM, Gandhi L, Riely GJ, et al. Safety and activity of alectinib against systemic disease and brain metastases in patients with crizotinib-resistant ALK-rearranged non-small-cell lung cancer (AF-002JG): results from the dose-finding portion of a phase 1/2 study. Lancet Oncol 2014;15:1119-28.

29. Katayama R, Sakashita T, Yanagitani N, et al. 
P-glycoprotein Mediates Ceritinib Resistance in Anaplastic Lymphoma Kinase-rearranged Non-small Cell Lung Cancer. EBioMedicine 2016;3:54-66.

30. Kodama T, Hasegawa M, Takanashi K, et al. Antitumor activity of the selective ALK inhibitor alectinib in models of intracranial metastases. Cancer Chemother Pharmacol 2014;74:1023-8.

31. Zhou C, Kim SW, Reungwetwattana T, et al. Alectinib versus crizotinib in untreated Asian patients with anaplastic lymphoma kinase-positive non-small-cell lung cancer (ALESIA): a randomised phase 3 study. Lancet Respir Med 2019;7:437-46.

32. Ito K, Hataji O, Kobayashi H, et al. Sequential Therapy with Crizotinib and Alectinib in ALK-Rearranged NonSmall Cell Lung Cancer-A Multicenter Retrospective Study. J Thorac Oncol 2017;12:390-6.

33. Asao T, Fujiwara Y, Itahashi K, et al. Sequential Use of Anaplastic Lymphoma Kinase Inhibitors in Japanese

Cite this article as: Pan Y, Xiao W, Ye F, Wang H, Shen Y, Yu X, Han X, Chu Q, Zhou C, Zhang Z, Ren S. Outcomes of switching from crizotinib to alectinib in patients with advanced non-small cell lung cancer with anaplastic lymphoma kinase fusion. Ann Transl Med 2021;9(12):1014. doi: 10.21037/atm21-2769
Patients With ALK-Rearranged Non-Small-Cell Lung Cancer: A Retrospective Analysis. Clin Lung Cancer 2017;18:e251-8.

34. Novello S, Mazières J, Oh IJ, et al. Alectinib versus chemotherapy in crizotinib-pretreated anaplastic lymphoma kinase (ALK)-positive non-small-cell lung cancer: results from the phase III ALUR study. Ann Oncol 2018;29:1409-16.

35. Ito K, Yamanaka T, Hayashi H, et al. Sequential therapy of crizotinib followed by alectinib for non-small cell lung cancer harbouring anaplastic lymphoma kinase rearrangement (WJOG9516L): A multicenter retrospective cohort study. Eur J Cancer 2021;145:183-93.

36. Zhu V, Ou SH. Safety of alectinib for the treatment of metastatic ALK-rearranged non-small cell lung cancer. Expert Opin Drug Saf 2017;16:509-14.

(English Language Editor: J. Reynolds) 